\title{
THE EFFECTS OF EARTHQUAKES ON BUILDINGS WITH A FLEXIBLE FIRST STORY ${ }^{1}$
}

By R. R. MARTEL 2

The title of this paper is a misnomer, purposely selected to indicate the reason for its preparation rather than its contents. It is not proposed to consider the effects of the extremely complex motions of earthquakes on such a complex structure as the ordinary building, but rather to idealize both the motion and the structure in order to obtain some basic concepts which may perhaps have bearing on the general topic suggested by the title. The scope of the paper then will be limited to a consideration of but one bent of a building subjected to established simple harmonic motion.

It has been suggested ${ }^{3}$ that steel frame buildings might be designed successfully to withstand earthquakes by the use in their first story of long and relatively flexible columns with walls made intentionally weak so as to offer but slight resistance to the bending of the first story columns, while in the upper stories, the ordinary framing and the walls would be relatively so stiff that substantially all the deflection caused by horizontal movements of the ground would take place in the first story. As a first approximation, such a structure may be assumed to be like a one-story bent, and its behavior in an earthquake may be somewhat similar to that produced by established simple harmonic motion. For this idealized case, expressions have been derived in the appendix which give $(a)$ the natural free period of the bent and $(b)$ the maximum amplitude of the top of the bent relative to the maximum amplitude of the applied motion of the base in terms of the ratio of the free period of the bent to the period of the applied motion of the base.

1 Presented before the Seismological Society of America, at Berkeley, California, on June 20, 1929.

2 Associate Professor of Civil Engineering, California Institute of Technology, Pasadena, California.

${ }^{3}$ L. H. Nishkian, "Design of Tall Buildings for Resistance to Earthquake Stresses," The Architect and Engineer, 88, 73, March 1927; and C. H. Snyder, "Earthquakes and Building Codes," Engineering Neres-Record, 98, 995, June 16, 1927. 
To form some idea of the advantages and limitations of the flexiblefirst-story type of construction, studies have been made of the action of a few single-story bents with columns and loads so selected as to cover a fairly wide range of conditions that might be expected to occur. The results of these studies are summarized in Tables I and II.

In Table I, Column 1 gives the size and weight of some Bethlehem rolled column sections. The length of the column given in Column 2 was taken so that the value of the slenderness ratio, $L / r$, was 120 . In computing the natural period, Column 3 , it was assumed $(a)$ that the columns were carrying the maximum allowable load according to the A.I.S.C. column formula (viz., 10,000 pounds per square inch times the area of the column cross-section), and $(b)$ that the columns were direction-fixed at both ends. Then the general expression for the period,

becomes

$$
T=2 \pi \sqrt{\frac{m h^{3}}{12 E I}}
$$

$$
T=2 \pi \sqrt{\frac{10,000 A h^{3}}{12 E A r^{2} g}}
$$

or, if $r$, the radius of gyration, is in inches and $h$, the length, is in feet,

$$
T=0.070 \frac{h}{r} \sqrt{h}
$$

The radius of gyration that has been used in computing the free period was that about an axis perpendicular to the web of the columns. It is obvious that the effect of fire-proofing on the period has not been taken into account, the assumption being that the material used for this purpose, say gypsum blocks, would have but little strength and very low modulus of elasticity.

The values for the permissible deflection given in Column 4 are those which would produce a combined stress of 27,000 pounds per square inch in the columns. This stress is the sum of the direct stress, $f^{\prime}$, due to the vertical load, the bending stress due to eccentricity of the vertical load when the column is deflected, and the bending stress due to the horizontal shear. The bending moment from the latter increases directly as a straight line from the point of inflection. Though the bending moment due to eccentricity does not vary as a straight line, it adds but little to 


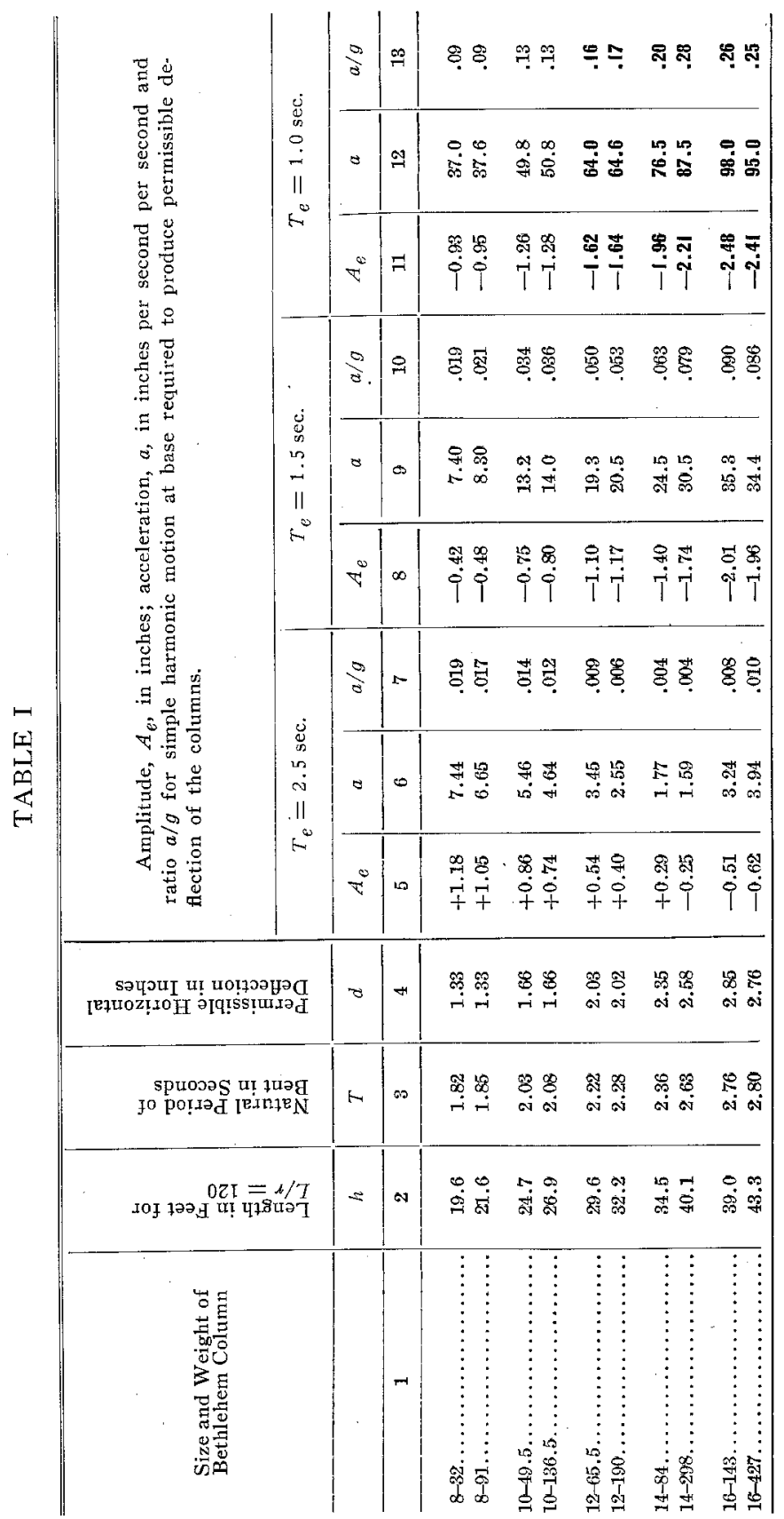


the deflection caused by shear and may, without appreciable error, be taken as varying as a straight line.

If $M$ is the sum of the moments due to the shear and eccentricity then

but

$$
d=\frac{M h^{2}}{6 E I}
$$

hence

$$
M=\frac{f^{\prime \prime} I}{c}
$$

and since

$$
d=\frac{f^{\prime \prime} h^{2}}{6 E c}
$$

then

$$
f^{\prime \prime}=27,000-f^{\prime}=17,000
$$

$$
d=\frac{17,000 h^{2}}{6 E c}
$$

If the half depth of the section, $c$, is expressed in inches, $h$ in feet, and $E$ is taken at 30,000 kips per square inch,

$$
d=\frac{0.0136 h^{2}}{c} \text { inches. }
$$

It will be noted that, in the preceding discussion, the effect of the direct stress in the columns due to overturning has been neglected. This stress would depend on the height of the building and, for tall buildings, its inclusion would materially reduce the permissible deflections which have been computed in Tables I and II. Thus the range of periods for which flexible first-story columns are effective would be narrowed. However it seemed best not to include the consideration of these overturning stresses in the present discussion because the numerous additional tables that would be required would detract from the simplicity of the treatment.

The amplitude of the simple harmonic motion of the base, $A_{e}$, which will cause for a given period, $T_{e}$, the column deflection $d$ may be obtained by recasting the general expression given in the appendix.

$$
d=A_{1}-A_{e} \quad A_{1}=A_{e} \frac{1}{1-\left(\frac{T_{1}}{T_{e}}\right)^{2}}
$$




$$
\begin{aligned}
& d=A_{e}\left[\frac{1}{1-\left(\frac{T_{1}}{T_{e}}\right)^{2}}-1\right] \\
& A_{e}=d\left[\left(\frac{T_{e}}{T_{1}}\right)^{2}-1\right]
\end{aligned}
$$

The values of $A_{e}$ for certain assumed values of the period of the motion applied at the base are given in columns 5,8 , and 11 . The accelerations corresponding to the amplitudes and periods have been computed from the relation

$$
a=4 \pi^{2} \frac{A}{T^{2}}
$$

and the results, expressed in inches per second, are given in columns 6 , 9 , and 12 , and the values of the ratio of the acceleration $A_{e}$ to that of gravity are given in columns 7,10 , and 13 .

Positive values of $A_{e}$ indicate that the top of the bent moves in the same direction as the base. Negative values of $A_{e}$ indicate that the top of the bent moves in a direction opposite to that of base; i.e., there is a phase difference of 180 degrees in the motions of the top and bottom.

In obtaining the data for Table II (p. 172) the procedure was quite similar to that outlined above for Table I, but, instead of taking the column length at 120 times the least radius of gyration, lengths of twenty and twenty-four feet were used for each column. The allowable stress then for direct load varies with the length, and the mass at the top of the bent is dependent on this stress. The natural period,

$$
\begin{aligned}
& T=2 \pi \sqrt{\frac{m h^{3}}{12 E I}} \\
& T=2 \pi \sqrt{\frac{f^{\prime} A h^{3}}{12 A r^{2} g}}
\end{aligned}
$$

or if $f^{\prime}$ is in kips per square inch, $h$ in feet and $r$ in inches

$$
T=0.0221 \frac{h}{r} \sqrt{f^{\prime} h}
$$




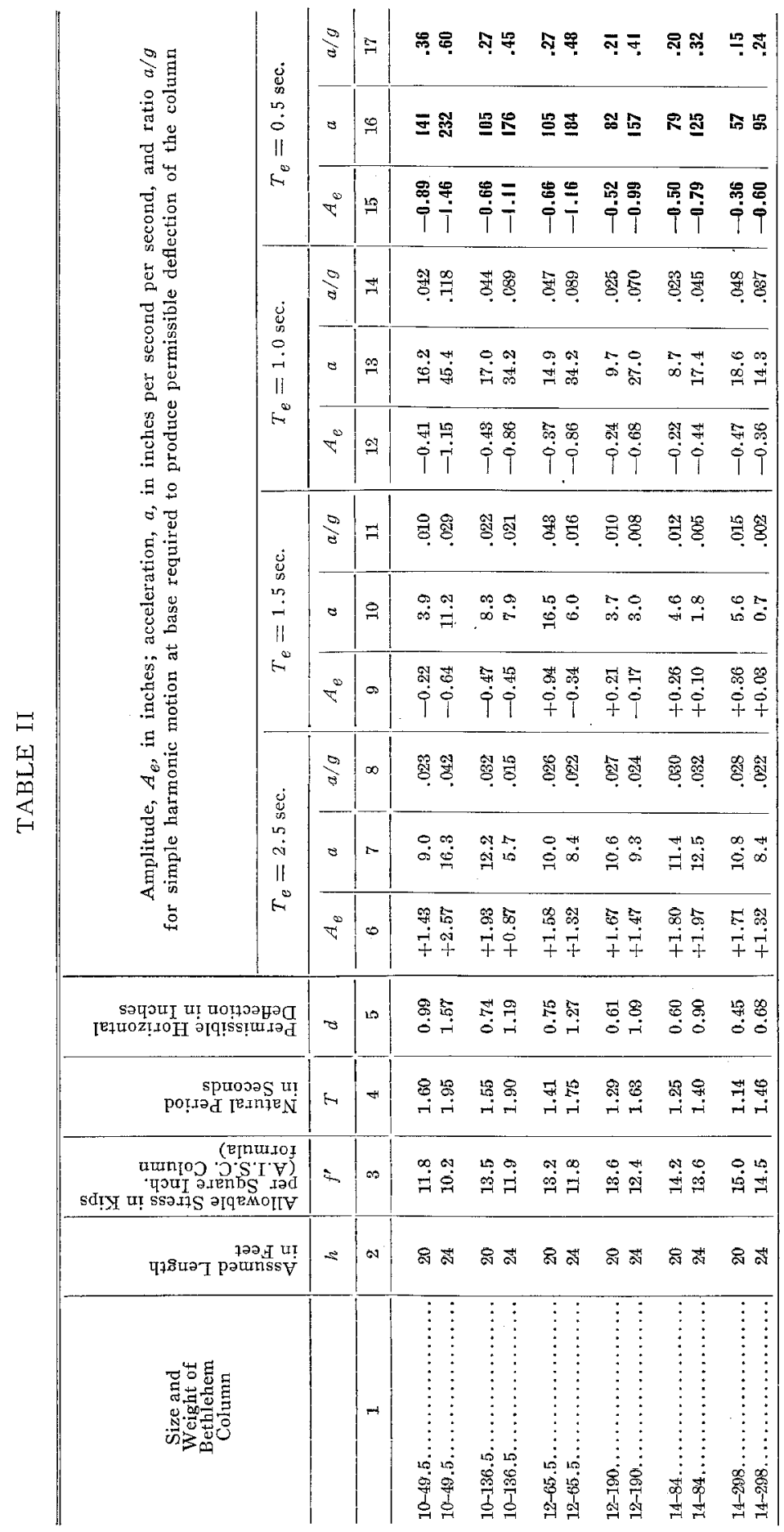


To find the permissible deflection for a combined stress just equal to 27,000 pounds per square inch we have

$$
f^{\prime}+\frac{6 E c d}{h^{2}}=27,000 \text { pounds to the square inch }
$$

Expressing $c$ in inches, $h$ in feet, and $f^{\prime}$ in kips, the deflection in inches

$$
d=\left(27-f^{\prime}\right) \frac{h^{2}}{1250 c}
$$

These are the only modifications of the expressions worked out for Table I that are needed in order to compute the data in Table II.

Since the amplitude, $A_{1}$, of the top of the bent is equal to the algebraic sum of the amplitude, $A_{e}$, and the deflection, $d$, it follows that when $A_{e}$ is positive the amplitude and consequently the acceleration of the top of the bent will be greater than the amplitude and the acceleration of the base respectively. For such cases the flexible first-story columns fail to accomplish their intended purpose of reducing the acceleration of the top. Since with this type of construction it is not proposed to use any special stiffening in the upper stories, it would seem proper to say that when the acceleration transmitted to the top exceeds some fraction, say twenty-five per cent, of the acceleration of the applied motion, the effectiveness of the method ceases.

Moreover, those cases, where the acceleration of the base necessary to stress the columns to 27,000 pounds per square inch is less than some value arbitrarily fixed at say $0.1 \mathrm{~g}$, should also be excluded as beyond the range of applicability of the method.

Then, the limits of successful performance are

$$
A_{e}+d<\frac{A_{e}}{4}
$$

$$
\frac{a}{g}>1
$$

The cases lying within these limits have been underlined in Tables I and II.

A question may be raised regarding the validity of the assumption that the dynamic behavior of a multi-story building with flexible first- 
story columns is similar to that of a single-story bent. Analytical studies of simple bents of two, three, and four stories have been made and relations obtained between the ratio of the amplitude of the various floors to that of the base and the ratio of the natural period to the period of the applied motion. These analytical results, when plotted for the case of a four-story bent with first-story columns ten times as flexible $e^{4}$ as those in the upper stories, give curves.which lie close to that obtained by plotting the equation for a one-story bent, except for sharp departures at values of $\frac{T_{1}}{T_{0}}$ of 5,9 , and 12 . These sharp departures result from the fact that a bent has just as many modes of vibration as there are stories. Hence, for any of the periods of the applied motion corresponding to these modes of vibration, resonance may occur. Unfortunately, since these periods are shorter than the fundamental, they lie within the range of periods where the long flexible first columns appear effective when considered as a single-story bent.

While it may be that harmonic resonance at these periods is not likely to occur in buildings because of the sharpness of tuning required and the rapid damping, we have as yet no assurance that such is the case. They have been left out of consideration in the preceding analysis merely to simplify and generalize the treatment.

If the dynamic behavior of a building with flexible first-story columns which is subjected to an earthquake is similar to that of a one-story bent under established simple harmonic motion, the following conclusions may be drawn from the foregoing discussion and the data in Tables I and II :

1. The flexible first story does not reduce the accelerations of the upper portions of the building when the period of the earthquake is greater than the free period of the building. For such cases the greater the flexibility, the greater the acceleration of the upper portion.

2. The limits of successful performance of the flexible first story are fixed, not only by the permissible deflection of the columns, but by the acceleration transmitted to the upper portion of the building. For the most favorable cases which have been considered, cases which would be difficult to realize in practice, these limits are for earthquakes with periods of one second or less.

3. Even in this range the possibility of harmonic resonance remains.

\footnotetext{
4 As used here this means that $\frac{h_{1}{ }^{3}}{I_{1}}=\frac{10 h_{2}{ }^{3}}{I_{2}}$
} 


\section{APPENDIX}

\section{Dynamic Behayior of a One-Story Bent Subjected to Simple Harmonic Motion}

The relation derived below gives the amplitude of motion of the top of a one-story, single-span bent relative to the amplitude of the horizontal simple harmonic motion applied at the base of the bent in terms of the ratio of the free or natural period of the bent to the period of the applied motion. The result is the classic equation for an undamped seismograph, but the method of its derivation, which is believed to be new, is simpler than those methods which are based on the use of differential equations.

The assumptions that have been made in this derivation are:

1. That the base is subjected to established simple harmonic.

2. That the mass is concentrated at the top, the weight of the supports being neglected.

3. That there is no damping.

4. That the columns are direction-fixed at both ends.

Nomenclature:

$A_{0}=$ maximum amplitude of base.

$A_{1}=$ maximum amplitude at top of bent.

$a=$ maximum horizontal acceleration.

$g=$ acceleration of gravity 32.2 feet per second per second $=386$ inches per second per second.

$d=$ maximum horizontal deflection of top of bent with reference to the base.

$h=$ height of bent.

$m=w / g=$ mass at the top of the bent.

$F=$ maximum horizontal force at top of bent produced by acceleration.

$I=$ amount of inertia of one column.

$E=$ modulus of elasticity of the columns.

$T_{0}=$ period of applied motion.

$T_{1}=$ free (natural) period of the bent.

$a_{1}=$ maximum acceleration of top of bent.

The Free Period, $T_{1}$

For the case shown in Figure 1 (p. 176), which shows the bent when the floor is at its maximum displacement,

$$
d_{1}=\frac{F h^{3}}{12 E I}
$$




$$
=F c^{*} \quad \text { in which } c=\frac{h^{3}}{12 E I}
$$

but

$$
F=m a_{1}
$$

and

$$
a_{1}=4 \pi^{2} \frac{A_{1}}{T_{1}^{2}} \text { for simple harmonic motion }
$$

or.

then

$$
a_{1}=4 \pi^{2} \frac{d_{1}}{T_{1}^{2}} \text { since } A_{1}=d_{1}
$$

hence

$$
d_{1}=4 \pi^{2} m c \frac{d_{1}}{T_{1}^{2}}
$$

$$
T_{1}^{2}=4 \pi^{2} m c
$$

so

$$
T_{1}=2 \pi \sqrt{\frac{m h^{3}}{12 E I}}
$$

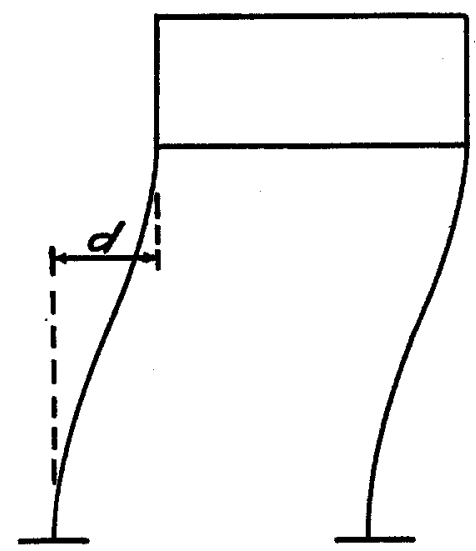

FIG. 1

Amplitude of ToP Under Forced Vibration

The two possible conditions of distortions are shown in Figure $2(a)$ and $(b)$, and the equations which follow apply equally well to each case.

* See footnote 5 on page 178. 


$$
\begin{aligned}
d_{1} & =F_{c} \quad\left(c=\frac{h^{3}}{12 E I}\right) \\
& =m a_{1} c \\
& =4 m c \pi^{2} \frac{A_{1}}{T_{0}^{2}} \\
& =T_{1}^{2} \frac{A_{1}}{T_{0}^{2}} \\
A_{0} & =A_{1}-d_{1} \quad A \text { being positive to the right of } 0 \\
A_{0} & =A_{1}\left(1-\frac{T_{1}^{2}}{T_{0}^{2}}\right) \\
\frac{A_{1}}{A_{0}} & =\frac{1}{\left(1-\frac{T_{1}^{2}}{T_{0}^{2}}\right)}
\end{aligned}
$$
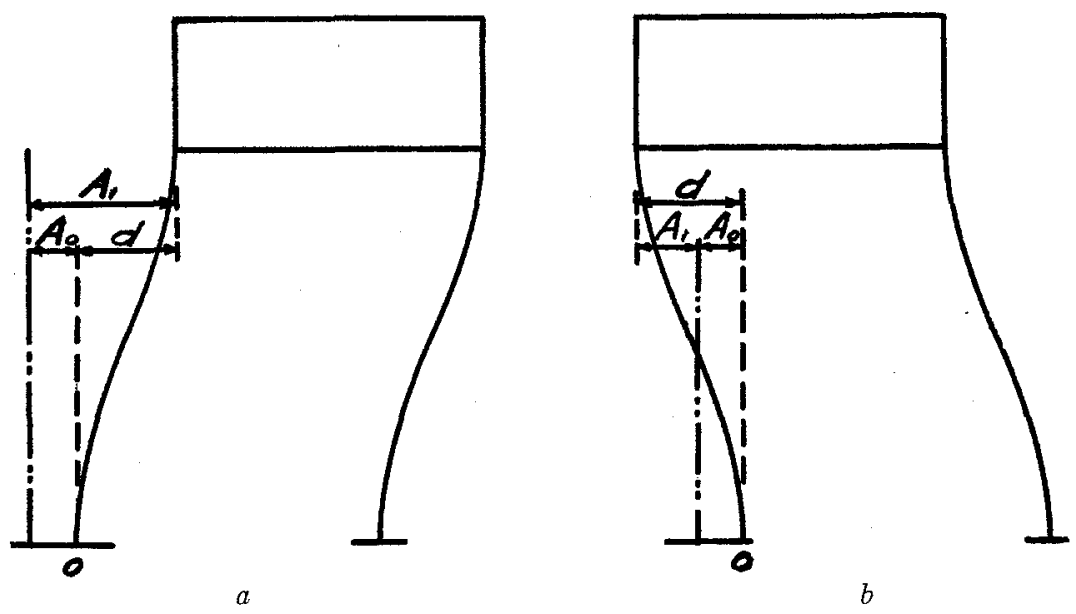

Fig. 2

This last equation is dependent on three and only three variables, but the weight of the floor and stiffness of supports enters in $T_{1}$. This relation is, however, quite general and may be used for any system in which the force required to produce a displacement is directly proportional to the displacement. In cases where more than one column or more than 
one bent are involved the only change necessary in the foregoing treatment is in the determination of $T_{1}$. This can readily be done by finding the value of the deflection produced by a unit horizontal force ${ }^{5}$ acting on the structure as a whole instead of on a two-column bent.

5 It should be noted that if $F$ is unity, $c=d_{1}$ or $c$ may be defined as the deflection produced by a unit force. 